\title{
Method developed for mass production of nanofibers
}

TECHNOLOGY ADVANCES seeks materials developments

on the threshold of commercialization.

Send suggestions to Renée G. Ford,

Renford Communications, renford@comcast.net.

\section{The pitch}

Nanofibers, defined as fibers with diameters of $<100 \mathrm{~nm}$ to $<500 \mathrm{~nm}$, are desirable enhancements for a number of promising applications including medical, filtration, energy, textile, protective, structural, electrical, and optical. However, their commercial potential has not yet been realized because the limitation in the scalability of present production technologies restricts their application to relatively high-priced niche products. Forcespinning ${ }_{\text {тм }}$ developed by FibeRio Technology expands the range of materials that can be made into nanofibers to include polymers, metals, ceramics, and composites while meeting mass production costs and productivity requirements with an environmentally sound approach. FibeRio recently launched the Cyclone L-1000M laboratory equipment to enable research and development in Forcespinning nanofibers, as industrial production equipment is rapidly developed.

Current market research estimates that the nanofiber market will reach $\$ 2.2$ billion over the next 10 years. Industrial-

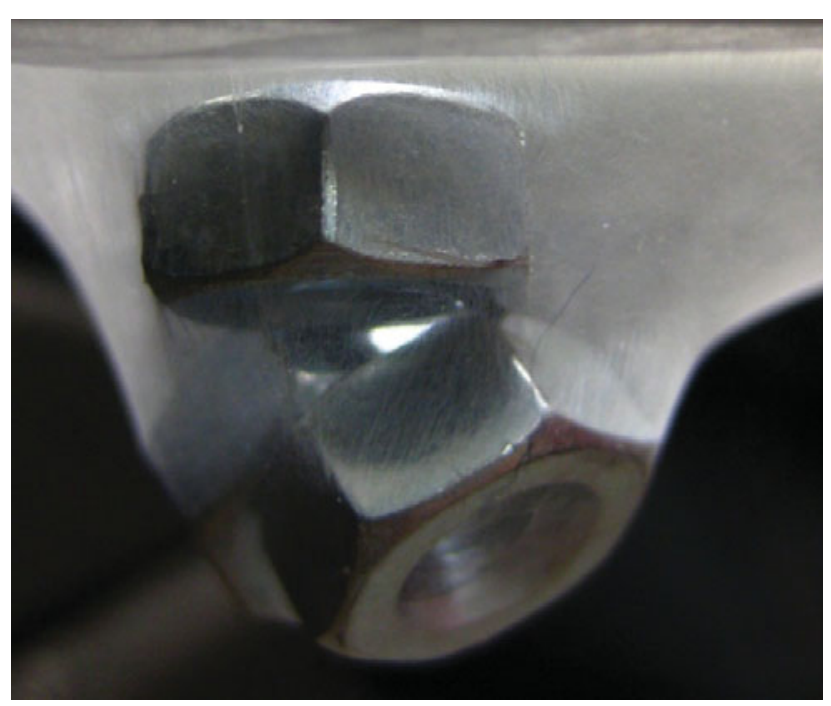

Unsupported polyethylene oxide (PEO) nonwoven nanofiber thin mat bearing added weight to show structural integrity (strength and strain) needed for biomedical applications. scale Forcespinning is anticipated to expedite this goal by impacting the market from two significant aspects: substantial cost reduction, which should make it possible for the filtration market (the largest current market application) to greatly enhance its utilization of nanofibers plus new applications that will be enabled by Forcespinning's versatility. The broader range of materials made possible by the use of centrifugal force plus FibeRio's strategy of providing laboratory products for academia and research and development $(R \& D)$ is expected to expand the reach of feasible nanofiber applications into technical textiles, ultracapacitors, catalysis, battery separators, tissue engineering, wound care, drug delivery, aerospace, and solar.

\section{The technology}

Forcespinning produces nanofibers using specially designed spinnerets in combination with centrifugal force and thermal, speed, and collection systems. The use of centrifugal versus electrostatic force to draw fibers eliminates the constraints of dielectric requirements for solvents and processed materials, greatly expanding the range of materials that can be processed into fibers. The ability to melt and spin molten materials provides a $100 \%$ yield increasing output and reducing solvent purchase cost and hazardous material recovery costs. At the industrial scale, Forcespinning has the potential to cost only one quarter of the cost of alternative technologies.

Forcespinning also has the addi- tional advantage of easily controlling fiber morphology through the adjustment of spinneret rotational speed, orifice shape, and distance to the collector. Alternative processing techniques for nanofiber development such as template synthesis, phase separation, self-assembly, melt blowing, and electrospinning all have limitations in their ability to satisfy industrial-scale requirements as well as their capability of attaining targeted fiber morphologies. Electrospinning, the current leading commercial technology, has several disadvantageous properties that severely affect fiber formation. These include solution or melt conductivity, electrostatics, electric field strength, surface charge, and ionization field. In the case of the melt blowing process, the requirement for hot air at supersonic speed (to create $<500 \mathrm{~nm}$ nanofibers) is a limiting factor for industrial production. Therefore, to date, electrospinning has been considered the only method with the potential for industrial production.

In addition to being a flexible system, Forcespinning offers the potential for the economic mass production of a broad range of polymeric, metallic, ceramic, and composite fibers in the nano-, submicrometer, and single digit micrometer scale. The collection system allows flexibility to readily collect material either as free-standing nonwoven webs (shown in Figure) for applications such as scaffolds for tissue engineering or deposited onto substrates for filtration. When collected as webs, the fibers can also be spun into yarns that can be woven into many desired textile applications.

\footnotetext{
Opportunities

FibeRio is evaluating opportunities for comaterials development and integrating pilot production and coating equipment into commercial partners' substrate production lines in nonwoven, textiles, and filtration industries. Source: Kial Gramley, FibeRio Technology Corporation, 501 N. Sugar Road, Edinburg, TX, USA; tel. 956-207-5448; e-mail info@ fiberiotech.com; and www.fiberiotech.com.
} 